\title{
A Series Chain-Link Modular Multilevel DC-DC Converter For High Voltage and High Power Applications
}

\author{
Beeond M. Saleh, Alessandro Costabeber, Alan J. Watson, Jon C. Clare \\ Power Electronics, Machines and Control Research Group \\ University of Nottingham \\ Nottingham, United Kingdom \\ Beeond.M.Saleh@nottingham.ac.uk
}

\begin{abstract}
In high voltage and high-power applications, many modular multilevel DC-DC converter topologies have been proposed for DC voltage scaling applications. In most of those proposed a three phase transformer is required. The construction of a three-phase transformer at high power and high voltages can be challenging. For example, in multi-terminal HVDC applications, several hundred kilo volts of isolation may be required. Additionally, a high number of submodules is required in most of the high voltage modular multilevel DC-DC converters. In this paper, a new modular multilevel DC-DC converter is proposed for high voltage applications, which aims to significantly reduce the number of submodules and achieve a more compact stored energy distribution. Furthermore, a number of lower voltage rated transformers are used to replace a single high voltage component. A simulation system for a $10 \mathrm{MW}, 20 \mathrm{kV}-20 \mathrm{kV}$ Series Chain-Link DC-DC converter, simulated in PLECS to verify the proposed topology.
\end{abstract}

Index Terms-High voltage DC-DC power converter, DC transformer for HVDC, modular multilevel converter (MMC)

\section{INTRODUCTION}

Modular Multilevel Converter schemes for high voltage and high power DC-DC conversion have been intensively addressed by the researchers in recent years to firstly, fulfil the requirements of high voltage DC transmission systems where isolating systems at the same or different voltages are crucial [1], [2]. Secondly, high voltage and high power DCDC converters have been studied in literature to integrate large scale renewable energy, wind farms and solar power stations, into HVDC systems where in some cases high voltage gain DC-DC converters are required [3]-[5].

In the last decade several high voltage multilevel converters have been proposed and the most promising topologies for the mentioned applications that can be found in the literature are the following: Alternate Arm AAC-DC-DC converter, sinusoidal operation MMC-DC-DC converter, trapezoidal operation MMC-DC-DC converter, Controlled Transition Bridge converter and high gain MMC-DC-DC converters for interfacing some renewable energies with HVDC systems [3], [5]-[8].

In HVDC systems and high voltage DC networks such as, multi-terminal HVDC systems, the voltage levels are hundreds of kilo volts and the power rating is several hundreds of MW. For all of the aforementioned modular multilevel topologies either one three phase transformer or one single phase transformer is required. Building a cooling system at a high voltage and high power rating is challenging since the performance of the cooling system is important for prevention of the lifetime reduction of a transformer and this system also impacts the cost and the size of the transformer [9]. There are other reasons which make building a transformer at a rating of hundreds of kilo volts may not be practical, however, they will not be discussed further since it is not the main aim of this paper. In addition to the MMC based DC-DC converters there are a number of non MMC based DC-DC converter structures in literature. The cascaded multi DAB converter was proposed in [10] for HVDC applications. However, it may be practically difficult to implement as a result of the non modularity of the structure and the requirement of a high number of elementary cells to reach the required high voltages. Each of these cells comprises a low voltage single phase transformer and either a full-bridge or half-bridge converter. Accordingly, the MMC structures are more preferable for HVDC applications. Moreover, for such high voltage and high power applications and due to device voltage ratings, several hundreds to a few thousands of submodules (SM), based on system voltage rating, are required in building each of the proposed high voltage modular multilevel DC-DC converter topologies. Therefore, developing a modular multilevel DCDC converter that requires firstly, lower voltage and power rated transformers instead of a single high voltage and high power transformer and secondly, a significant reduction in the required submodules as compared to other proposed topologies are the main reasons for proposing a series connected chainlink DC-DC converter (SCC-DC-DC).

\section{PRinCiple OPERATION OF SCC-DC-DC CONVERTER}

Fig 1 presents four phases of the proposed SCC-DC-DC converter with a fixed DC source on both sides of the converter. A 1:1 (n:turn ratio=1) single phase, medium frequency transformer is considered in the AC link for each phase. Each phase consists of two chain-links; a primary chain-link and a secondary chain-link, and each chain-link consists of a series 
connection of half bridge submodules. This series connection produces $\frac{1}{N_{\text {phase }}}\left(N_{\text {phase }}\right.$ is the number of the phases $)$ of DC side voltage and an $\mathrm{AC}$ voltage. Considering each side of the converter, the sum of the $\mathrm{AC}$ chain-links voltage must be zero, therefore the chain-links AC voltage are shifted by $\frac{2 \pi}{N_{\text {phase }}}$ degrees considering the phases from 1 to $N_{\text {phase }}$ as shown in Fig. 1. However, for an even number of phases, operation can be set up where half of the phases can have same AC voltage but out of phase by $180^{\circ}$ with the other half. In each phase, $L_{k P}$ and $L_{k S}$ represent the primary leakage inductance and secondary leakage inductance of the transformer in addition to the tank inductance, respectively. $C_{b P}$ and $C_{b S}$ are the primary and secondary DC blocking capacitors, respectively and they block the DC voltage produced by the primary and secondary chain-links, respectively. The applied voltage on the transformer will be only AC on both sides. As a result, only $\mathrm{AC}$ current passes through the transformer and by imposing the AC voltage of the chain-link of one side (primary or secondary) of the converter and controlling the amplitude and the phase of the AC voltage of the chain-link of the other side of the converter, power flow can be controlled. Additionally, the chain-link current comprises the DC current and the $\mathrm{AC}$ current which is circulating between primary and secondary chain-links in each phase. The proposed SCC-DCDC converter is derived from [11] which has been proposed for HVDC grid interconnection. In [11] the AC link tank comprises passive components, inductors and capacitors, and a full-bridge based chain-link in order to have full control of the power factor whereas in the SCC-DC-DC the control of reactive power is not required and therefore the full-bridge chain-link can be eliminated.

Considering operation of SCC-DC-DC shown in Fig. 1, assuming a lossless converter and a unity transformer turn ratio, the primary and secondary chain-link voltages, $V_{C h P_{i}}$ and $V_{C h S_{i}}$, can be expressed as shown equation 1 and equation 2 respectively where $M_{\text {index }}$ is the modulation index, $\omega$ is the angular frequency, $V_{D C P}$ and $V_{D C S}$ are the primary and secondary DC link voltages, respectively and $i=1, \ldots, N_{\text {Phase }}$ indicates the phases. Moreover, $V_{C h S_{i}}$ and $\theta$ are the amplitude of secondary $\mathrm{AC}$ voltage and the voltage angle respectively and these are controlled to manage the transfer of the power flow. The chain-link currents can be expressed using equation 3 where primary, $\mathrm{P}$, and secondary, $\mathrm{S}$, are represented by $x$ and $\hat{I_{a c \_} i}$ represents the amplitude of AC link current.

$$
\begin{aligned}
& V_{C h P_{-} i}(t)=\frac{V_{D C P}}{N_{\text {Phase }}}\left(1+M_{\text {index }} * \sin \left(\omega t+\frac{\pi i-\pi}{2}\right)\right) \\
& V_{C h S_{\_} i}(t)=\frac{V_{D C S}}{N_{\text {Phase }}}+V_{C h S_{-} i} * \sin \left(\omega t+\frac{\pi i-\pi}{2}+\theta\right) \\
& I_{C h x_{-} i}(t)=I_{D C x}+\hat{i_{a c_{-} i}} * \sin \left(\omega t+\frac{\pi i-\pi}{2}\right)
\end{aligned}
$$

The number of submodules in one chain-link, $N_{S M x_{-} i}$, and the total number of required submodules, $N_{S M_{-} T}$, in the SCCDC-DC converter can be calculated as shown in equation 4 where $V_{S M}$ represents the nominal voltage of submodule. In comparison to a three phase MMC-DC-DC converter proposed in literature [3], [6], [8], the SCC-DC-DC requires only $33 \%$ of the submodules which are required for MMC-DC$\mathrm{DC}$ converter. The total number of submodules in the MMCDC-DC converter can be calculated as shown in equation 5 . Furthermore, the number of the phases in the SCC-DC-DC can be increased as required and by increasing the number of the phases, the number of single transformer increases whilst the total number of submodules in the SCC-DC-DC will remain the same, keeping $V_{S M}$ fixed. Additionally, increasing the number of phases will lead to a reduction in the DC current ripple- further explanation is given in the simulation section. Accordingly, building high voltage DC-DC converters with several single transformers can overcome the challenge of building one three phase high voltage transformer and overcome the cooling challenging due to lower voltage rating and power transformer rating. For providing high voltage gain, the chain-links of one side of the converter can be connected in parallel however, this will not be considered in this paper.

$$
\begin{aligned}
N_{S M x_{-} i}= & \frac{2 * V_{D C x}}{N \text { phase } * V_{S M x}} \\
N_{S M_{-} T}= & \text { Nphase } *\left(\frac{2 * V_{D C P}}{N \text { phase } * V_{S M P}}+\frac{2 * V_{D C S}}{\text { Nphase } * V_{S M S}}\right) \\
= & \frac{2 * V_{D C P}}{V_{S M P}}+\frac{2 * V_{D C S}}{V_{S M S}} \\
& N_{S M_{-} M M C_{-} T}=6 *\left(\frac{V_{D C P}}{V_{S M P}}+\frac{V_{D C S}}{V_{S M S}}\right)
\end{aligned}
$$

\section{ENERGY STORAGE ANALYSIS OF SCC-DC-DC}

In this section some considerations are given to select the optimum resonant tank and chain-link energy storage. The chain-link energy variation, $\Delta e_{C h x}(t)$, can be derived by integrating the instantaneous chain-link power, $P_{C h x}(t)$, over the fundamental period as described in equation 6 . The instantaneous stored energy in the chain-links, $E_{C h x} \_i(t)$, can be described as in equation 8 considering ideal energy sharing between the submodules within the corresponding chain-link where $C_{S M}$ is the SM capacitance. Based on the expressions (6) and 8 the peak to peak energy variation can be limited by choosing a desired $C_{S M}$ value where $\delta_{p p}$ is the per unit peak to peak energy variation normalised with the nominal $V_{S M}$. Knowing the required $C_{S M}$ and the equation of the energy variation of the chain-link, the per phase stored energy, $E_{C h} i$ can be approximated as presented in equation 9 .

$$
\begin{gathered}
\Delta e_{C h x \_} i(t)=\int P_{C h x_{\_} i}(t) d t=\int V_{C h x_{\_} i}(t) I_{C h x_{\_} i}(t) d t \\
E_{C h x \_} i(t)=\frac{1}{2} C_{S M x} N_{S M x} V_{S M x}(t)^{2} \\
C_{S M x} \geq \frac{\Delta e_{p p \_} C h x}{N_{S M x} V_{S M x}^{2} \delta_{p p}}
\end{gathered}
$$




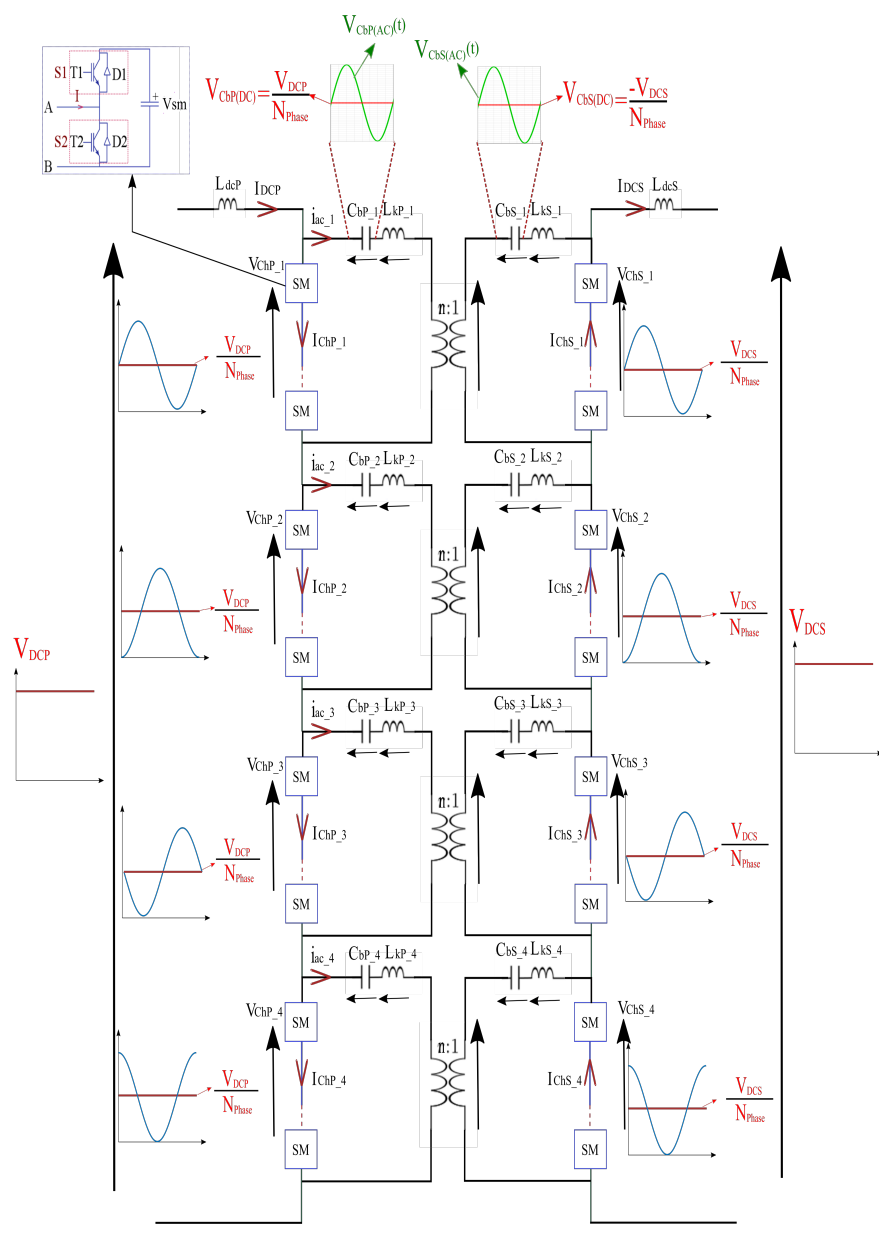

Fig. 1. SCC-DC-DC Converter

$$
E_{C h \_} i \approx \frac{\Delta e_{p p \_} C h P+\Delta e_{p p \_} C h S}{2 \delta_{p p}}
$$

Moreover, in order to evaluate the energy requirements of the tank, the peak voltage of the DC blocking capacitor, $V_{C_{b x \_}}$, is derived as in expression 10 where $X_{C_{b x}}=\frac{1}{\omega_{C_{b}}}$ is the impedance of the DC blocking capacitor. The stored energy for the per phase resonant tank, $E_{C L \_} i$ can be described as in expression 11 .

$$
\begin{gathered}
\hat{V_{C_{b x \_} i}}=\frac{V_{D C_{x}}}{N_{\text {phase }}}+\hat{i_{a c_{-} i}} * X_{C b x} \\
E_{C L \_} i=0.5 *{\hat{C_{b P}}} *{\hat{C_{b P \_} i}}^{2}+0.5 * C_{b S} * \hat{V}_{C_{b S \_} i}{ }^{2} \\
+0.5 * \hat{i_{a c_{-} i}{ }^{2}} *\left(L_{k P}+L_{k S}\right)
\end{gathered}
$$

In literature, generally the capacitance time constant, $H$, is used to express the required energy storage and its unit is $\mathrm{ms}$. The energy requirements, $H$, for SCC-DC-DC is presented in the expression 12 where $S$ is the apparent power of the converter.

$$
H=\frac{N_{\text {phase }} *\left(E_{C L \_} i+E_{C h \_} i\right)}{S}
$$

Assuming a unity transformer turns ratio, Fig. 2 shows the per phase AC equivalent circuit of the SCC-DC-DC where $L_{k}$ is the equivalent tank inductance, $L_{k}=L_{k P}+L_{k S}$, and $C_{b}$ is the equivalent tank capacitance, $C_{b}=\frac{C_{b P}}{2}=\frac{C_{b S}}{2}$. In order to show the optimum operating point where the minimum energy storage is required further considerations are required. The frequency factor, $K_{f}=\frac{f_{o}}{f_{r}}$, where $f_{o}$ is the operating frequency and $f_{r}$ is the resonant frequency between $L_{k}$ and $C_{b}$. The factor $Z_{b}=\frac{L_{k}}{L_{s}}$ where $L_{s}$ is the series inductance of the single phase transformer, assuming a $10 \%$ impedance. Assuming $\delta_{p p}=0.1 P U, M_{\text {index }}=1$ and by changing the factors $K_{f}$ and $Z_{b}$ with small steps from $0.55-1.6$ and $1-20$, respectively, the required per unit $H_{P U}=\frac{H}{H_{b}}$ is found at each point as presented in Fig. 3 where $H_{b}=\frac{1}{f_{o}}$ is the base capacitance constant. The optimum operating region is between $K_{f}=0.75-1.25$ and $Z_{b}=7-20$ where the $H_{P U}$ is between $6.14-6.3 P U$. However, operating at or below the resonant frequency of the LC tank makes the control over the AC current difficult. Therefore operating above resonance frequency is preferred and the $K_{f} \geq 1.15$ the minimum $H_{P U}$ is obtained when $Z_{b}=16$.

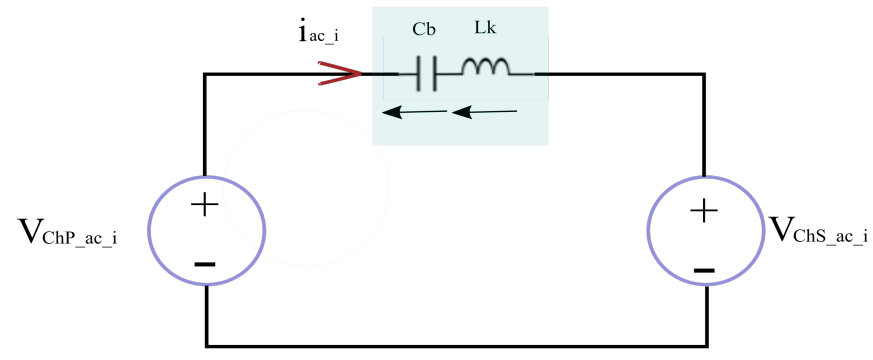

Fig. 2. Per phase equivalent circuit

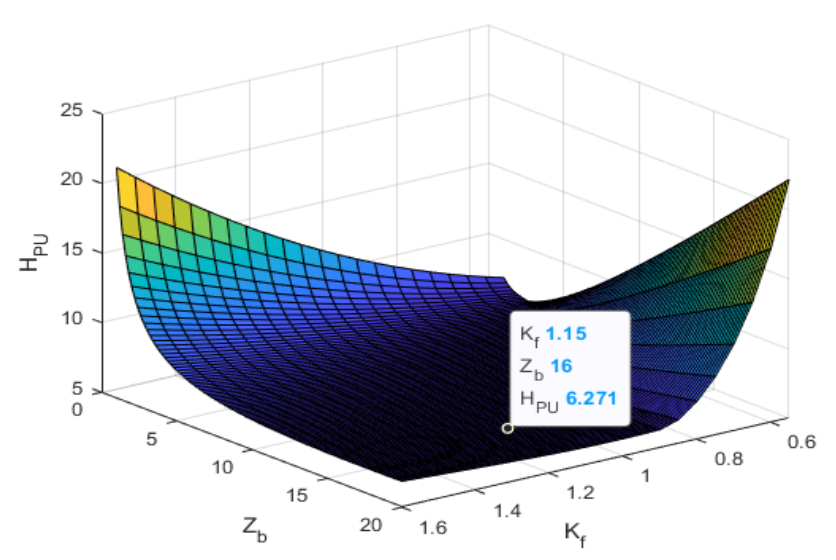

Fig. 3. Energy storage requirement analysis

In addition to a huge reduction in number of SMs, $66 \%$, and considering the same power rating the sinusoidal operation of SCC-DC-DC converter has less semiconductor losses in comparison to the sinusoidal operation of the MMC-DC-DC 
converter due to the absence of the second harmonic in the chain-link circulating current and the presence of resonant tank which filters ripple in the AC current of the SCCDC-DC. Regarding the energy storage, in literature the SMs capacitor energy storage for the MMC-DC-DC converter has been presented (excluding arm inductors and the effect of circulating current) and it requires around $75 \%$ of the energy storage required for the SCC-DC-DC. However, if the 12 arm inductors of the MMC-DC-DC and the second harmonic in the chain-link circulating current are considered, the required $H_{P U}$ for the MMC-DC-DC will be higher than $75 \%$ of the required $H_{P U}$ for the SCC-DC-DC.

\section{Control Structure of SCC-DC-DC}

This section shows the control schematic of the SCCDC-DC converter and the control loops that are necessary for the operation of the SCC-DC-DC are shown. The nonlinear differential function that describes the behaviour of each chain-link voltage can be linearised and approximated to the average voltage of the chain-link by using the transfer function 13 which describes the relation between the power and the average of the $\sum V_{S M x}$ of the chain-link, $V_{S M x_{-} e q}$. The power is imposed at the primary side of the SCC-DC-DC converter and per phase energy control is designed at the primary side to control the energy of each chain-link and provide the desired $\mathrm{AC}$ power reference for the inner per phase $\mathrm{AC}$ current control loop as shown in Fig. 4-a by using a PI compensator where $M e a$ represents the measured values and Ref represents the reference values.

$$
G_{E}(s)=\frac{V_{S M x \_e q}(s)}{P_{C h x}(s)}=\frac{1}{s V_{S M x} C_{S M x}}
$$
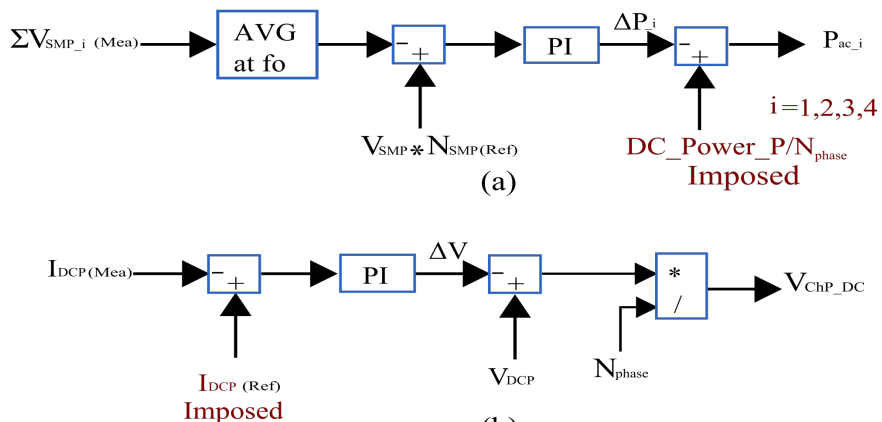

(b)

Fig. 4. (a) Primary energy control (b) Primary DC current control

The DC current controller is based on the transfer function shown in 14 which describes the relation between the DC current and the total DC voltages of the chain-links. Considering the primary side of the converter, $I_{D C P}(R e f)$ is imposed and by using a PI compensator the DC current is controlled and the DC component of the primary chain-links voltage reference is obtained as shown in Fig. 4-b.

$$
G_{D C x}(s)=\frac{1}{s L_{D C x}}
$$

The per phase AC link equation, 15, can be derived from Fig. 2 which was presented in previous section where only the AC voltage of the chain-links are considered since the DC voltages are perfectly blocked by $C_{b P}$ and $C_{b S}$ as previously explained. The per phase AC current control loop is the inner loop of per phase primary energy controller therefore it must be suitably "faster" than the outer energy control loop. A PR compensator with a complex zero at the resonant frequency of the $\mathrm{AC}$ resonant tank is designed to control the $\mathrm{AC}$ current and provide the $\mathrm{AC}$ voltage component of the secondary chainlink voltage references, $V_{C h S_{-} a c_{-} i}$, where the primary $\mathrm{AC}$ component of the chain-link voltage reference, $V_{C h P_{-} a c_{-} i}$, is imposed as shown in Fig. 5. a complex zero is used to cancel the effect of the complex poles of the resonant tank on the $\mathrm{AC}$ current response since the complex poles are close to the operating frequency due the design analysis with regards to the optimum energy requirements.

$$
V_{C h S \_a c \_i}(t)=V_{C h P \_a c_{-} i}-\frac{1}{C_{b}} \int i_{a c_{-} i}(t)-L_{k} \frac{d i_{a c_{\_} i}}{d t}
$$

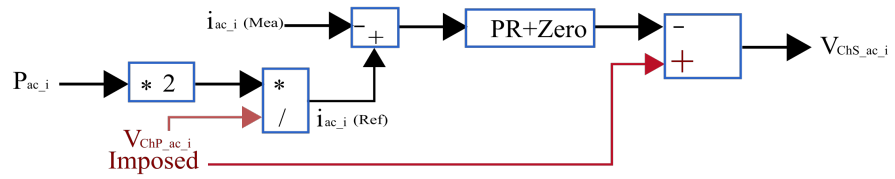

Fig. 5. Per phase AC link current control

For the secondary side of the converter, the total energy of the chain-link is controlled using a PI compensator as shown in Fig. 6-a and the same transfer function as in expression 13 describes the plant of the system. The total energy control of the secondary side of the converter provides the $I_{D C S_{-} r e f}$ to the inner DC current controller. Based on the same transfer function as in 14 a PI compensator is designed for the inner DC current loop to control the secondary DC current and provide the DC component of the secondary chain-links reference as shown in Fig. 6-b.

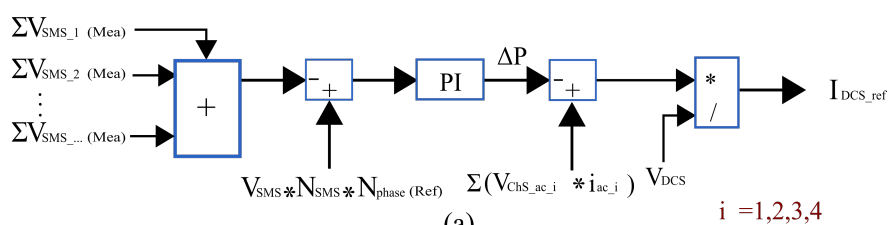

(a)

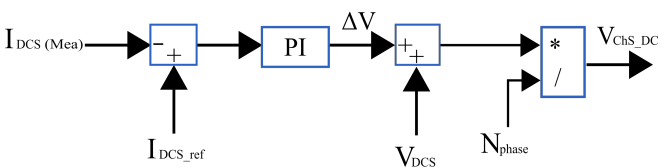

(b)

Fig. 6. (a) Secondary energy control (b) Secondary DC current control

The per phase energy controller at the primary side of SCCDC-DC keeps the energy stored at each chain-link of the 
primary side balanced during converter operation. However, having only the total energy controlled at the secondary side of SCC-DC-DC can result in the stored energy fluctuating between chain-links during operation. In order to maintain energy balance between the chain-links of the secondary side of SCC-DC-DC another control element is required. The energy balancing controllers shown in Fig. 7 provide the desired voltages that need to be added or subtracted to the reference of the chain-link voltages to equalize the energy storage of the secondary chain-links.

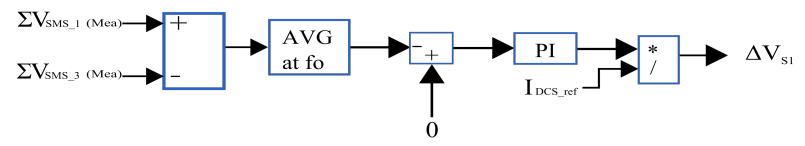

(a)

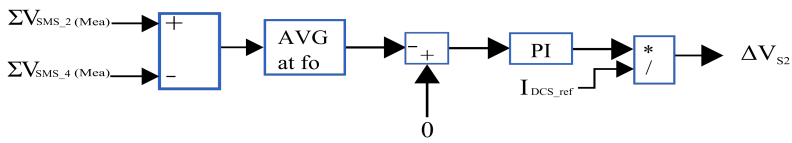

(b)

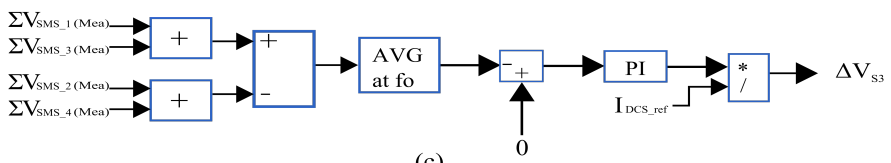

(c)

Fig. 7. Energy balancing between secondary chain-links

Fig.8-a and Fig.8-b,c show the per phase reference of the primary chain-links and the reference of the secondary chainlinks, respectively. The chain-links reference will be then compared with the carrier to provide the PWM signals to the SMs semiconductor devices of the chain-links.

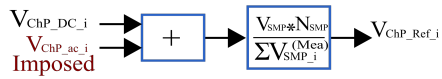

(a)
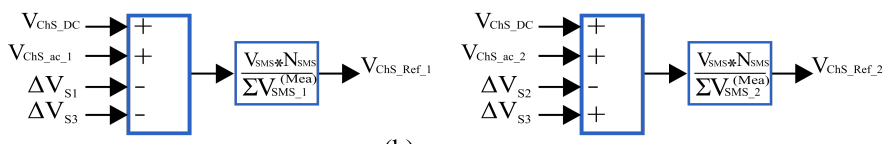

(b)
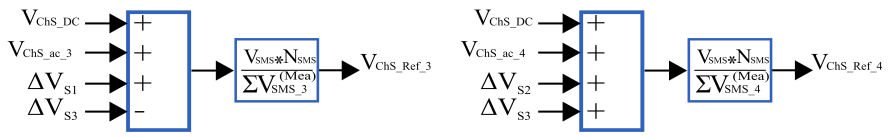

(c)

Fig. 8. (a) per phase primary chain-link reference voltage $(b, c)$ secondary chain-links reference voltage

\section{Simulation Results}

In order to validate the proposed topology, A $10 \mathrm{MW}$, $20 \mathrm{kV}-20 \mathrm{kV}$ four phase SCC-DC-DC converter model has been developed using PLECS where the $V_{C h x_{-} a c_{-} i}(t)$ are shifted by $\frac{\pi i-\pi}{2}$. The four phase SCC-DC-DC parameters are shown in Table I. Fig 9 shows the $\mathrm{AC}$ current and the passive components of the resonant tank voltages of phase A when the imposed DC power is changed from $10 M W$ to $8 M W$. It can be observed from Fig. 9-b,c that the $C_{b x}$ blocks the $V_{D C x} / N_{\text {phase. }}$. The capacitor SMs voltage control and balance of the primary and secondary chain-links at the nominal value $(1 \mathrm{kV})$ with $10 \%$ peak to peak ripple are presented in figure 10 .

TABLE I

Circuit PARAMEters

\begin{tabular}{|c|c|}
\hline Circuit Parameter & Value \\
\hline Primary DC Link Voltage $\left(V_{D C P}\right)$ & $20 \mathrm{kV}$ \\
\hline Secondary DC Link Voltage $\left(V_{D C S}\right)$ & $20 \mathrm{kV}$ \\
\hline AC Link Frequency $\left(f_{o}\right)$ & $250 \mathrm{~Hz}$ \\
\hline Transformer Turn Ratio $(n)$ & 1 \\
\hline Modulation index $M_{\text {index }}$ & 0.95 \\
\hline Submodule Capacitor $(C)$ & $5 \mathrm{mF}$ \\
\hline Submodule Capacitor Nominal Voltage $\left(V_{s m}\right)$ & $1 \mathrm{kV}$ \\
\hline Primary Submodule Number per phase $\left(N_{S M_{-} P}\right)$ & 10 \\
\hline Secondary Submodule Number Per phase $\left(N_{S M_{-} S}\right)$ & 10 \\
\hline Transformer Leakage Inductance $\left(L_{k P}=L_{k S}\right)$ & $4.6 \mathrm{mH}$ \\
\hline Capacitance of Blocking Capacitor $\left(C_{b P}=C_{b S}\right)$ & $0.23 \mathrm{mF}$ \\
\hline DC inductance $\left(L_{d c P}=L_{d c S}\right)$ & $1.3 \mathrm{mH}$ \\
\hline
\end{tabular}
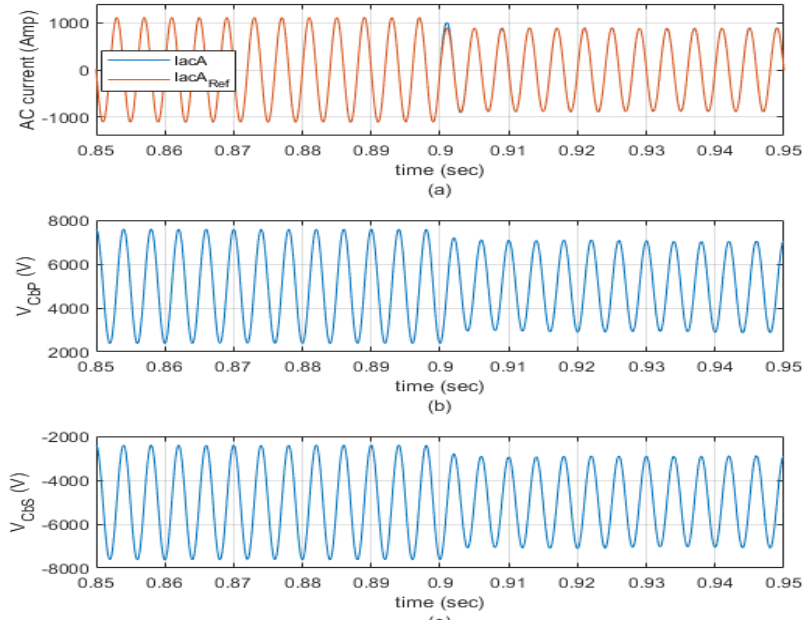

(c)

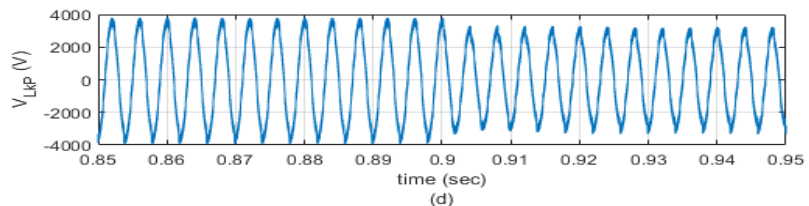

Fig. 9. (a) AC current response (b) Primary blocking capacitor voltage (c) Secondary blocking capacitor voltage (d) Primary inductor voltage

Furthermore, two operation methods have been tested to show the primary DC current response where as previously explained in the first operation method $V_{C h x_{-} a c_{-} i}(t)$ are shifted by $\frac{\pi i-\pi}{2}$. The second method is applicable only to a converter with even phases where the chain-link voltages for half of the phases ( 1 and 2 ) have the same phase, shifted by $\pi$ with respect to the other half (phases 3 and 4) and this will be similar to two phases. Considering the same change in power, 

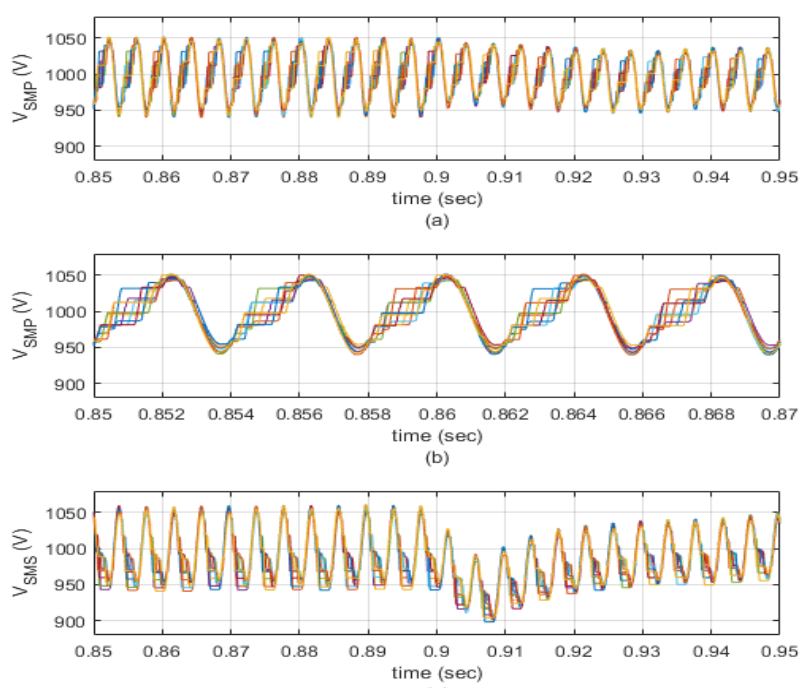

(c)

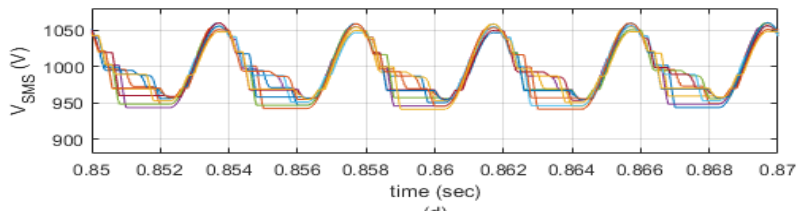

(d)

Fig. 10. (a) Primary SMs voltage (b) A small portion of primary SMs voltage (c) Secondary SMs voltage (d) A small portion of secondary SMs voltage

Fig. 11-a shows the DC current response for both operations where identical control schemes and converter parameters are chosen. It is clear that in Fig. 11-b the DC current of method $1, I_{d c P 1}$, has a small peak to peak ripple at $4 * f_{o}$, however, the method 2 DC current, $I_{d c P 1}$, has higher peak to peak ripple at $2 * f_{o}$. The reason for this is the harmonic order ripple in $\sum V_{C h x_{-} a c_{-} i}(t)$ where in second operation there is a second harmonic ripple in $V_{C h x_{a c} i}(t)$ whereas in first operation the harmonic order ripple, $H r_{o r}$, in $V_{C h x \_a c \_i}(t)$ depends on the number of the phases, $H r_{o r}=N_{\text {phase }} * f_{o}$. Consequently, with the first operational conditions, as the number of the phases increases the frequency of the ripple increases, and this provides the possibility to operate the converter at higher $\delta_{p p}$. As a result, this reduces the energy storage requirements for the converter. More mathematical analysis about the effect of increasing $N_{\text {phase }}$ on the transformer losses, converter losses and energy requirements are the subject of future work.

\section{CONCLUSION}

This paper proposed a SCC-DC-DC converter for highvoltage, high-power applications. The proposed topology is based on firstly, connecting chain-links in series to reduce the number of submodules and secondly, using a reasonable number of single phase transformers instead of one three phase transformer to overcome the associated high voltage and cooling system challenges. This paper has also shown the optimum

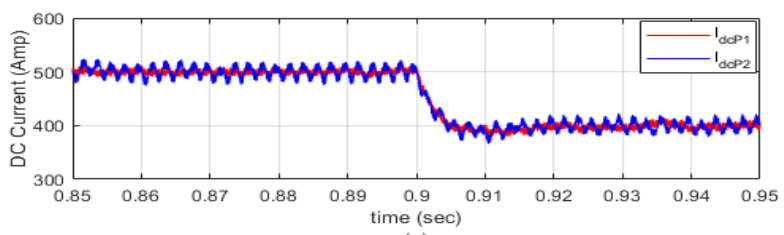

(a)

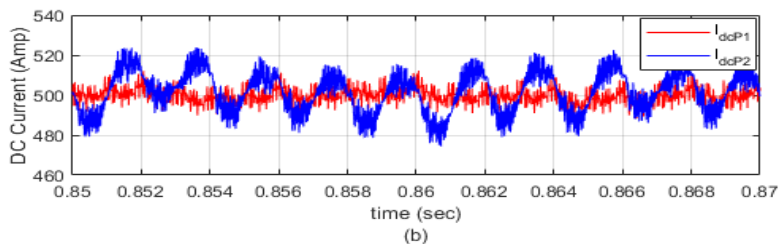

(b)

Fig. 11. (a) Primary DC current (b) A small portion of primary DC current

design region considering the energy storage requirements of the chain-links and resonant tank elements. The structure of the energy control loops, DC and AC control loops that are required to operate the SCC-DC-DC converter were presented in this paper. PLECS results show the effectiveness of the proposed topology and validate the control schemes for the proposed SCC-DC-DC converter.

\section{REFERENCES}

[1] X. Zhao and K. Li, "Adaptive backstepping droop controller design for multi-terminal high-voltage direct current systems," IET Generation, Transmission \& Distribution, vol. 9, no. 10, pp. 975-983, 2015.

[2] L. Zhang, Y. Zou, J. Yu, J. Qin, V. Vittal, G. G. Karady, D. Shi, and Z. Wang, "Modeling, control, and protection of modular multilevel converter-based multi-terminal hvdc systems: A review," CSEE Journal of Power and Energy Systems, vol. 3, no. 4, pp. 340-352, 2017.

[3] G. P. Adam, I. A. Gowaid, S. J. Finney, D. Holliday, and B. W. Williams, "Review of dc-dc converters for multi-terminal hvdc transmission networks," IET Power Electronics, vol. 9, no. 2, pp. 281-296, 2016.

[4] S. Kenzelmann, A. Rufer, M. Vasiladiotis, D. Dujic, F. Canales, and Y. R. De Novaes, "A versatile dc-dc converter for energy collection and distribution using the modular multilevel converter," in Power Electronics and Applications (EPE 2011), Proceedings of the 2011-14th European Conference on. Ieee, 2011, pp. 1-10.

[5] V. Chitransh and M. Veerachary, "A high-gain modular multilevel dc-dc step-up converter," in 2016 IEEE Uttar Pradesh Section International Conference on Electrical, Computer and Electronics Engineering (UPCON). IEEE, 2016, pp. 438-443.

[6] T. Lüth, M. M. Merlin, T. C. Green, F. Hassan, and C. D. Barker, "Highfrequency operation of a dc/ac/dc system for hvdc applications," IEEE Transactions on Power Electronics, vol. 29, no. 8, pp. 4107-4115, 2013.

[7] X. Zhang and T. C. Green, "The new family of high step ratio modular multilevel dc-dc converters," in 2015 IEEE Applied Power Electronics Conference and Exposition (APEC). IEEE, 2015, pp. 1743-1750.

[8] B. M Saleh, A. Costabeber, A. Watson, F. Tardelli, and J. Clare, "A new capacitor voltage balancing method for trapezoidal operation of modular multilevel dc-dc converters," in 201921 th European Conference on Power Electronics and Applications (EPE'19 ECCE Europe), Sep. 2019, pp. P.1-P.10.

[9] M. Djamali and S. Tenbohlen, "Malfunction detection of the cooling system in air-forced power transformers using online thermal monitoring," IEEE Transactions on Power Delivery, vol. 32, no. 2, pp. 1058-1067, 2016.

[10] M. J. Carrizosa, A. Benchaib, P. Alou, and G. Damm, "Dc transformer for dc/dc connection in hvdc network," in 2013 15th European Conference on Power Electronics and Applications (EPE). IEEE, 2013, pp. $1-10$.

[11] F. Tardelli, A. Costabeber, D. Trainer, and J. Clare, "Series chain-link modular multilevel ac-dc converter (scc) for hvdc applications," IEEE Transactions on Power Electronics, vol. 35, no. 6, pp. 5714-5728, 2019. 